\title{
Atuação do Enfermeiro em Cuidados Paliativos na Atenção Primária à Saúde: Revisão Integrativa
}

doi: https://doi.org/10.32635/2176-9745.RBC.2022v68n1.1383

\author{
Nursing Work in Palliative Care in Primary Health Care: Integration Review \\ Actuación del Enfermero en Cuidados Paliativos en la Atención Primaria a la Salud: Revisión Integrativa
}

\author{
Luan dos Santos Fonseca'; Beatriz Correia Carvalho' ${ }^{2}$; Héllen Oliveira Santos ${ }^{3}$; Jackeline Melo da Silva ${ }^{4}$; José Cleyton de Oliveira \\ Santos $^{5}$; Laíse Luemmy de Lima Ferreira ${ }^{6}$; Simone Yuriko Kameo ${ }^{7}$
}

\section{RESUMO}

Introdução: A atuação da enfermagem ao indivíduo em cuidados paliativos $(\mathrm{CP})$ na Atenção Primária à Saúde (APS) visa a promover a qualidade de vida dos indivíduos e da sua família como garantia da assistência integral, para um cuidado humanizado e digno, melhorando a maneira de enfrentar a doença e minimizando o sofrimento. Objetivo: Analisar e sintetizar a produçáo científica relacionada à assistência do enfermeiro ao indivíduo em CP nas APS. Método: Revisão integrativa da literatura realizada nas bases de dados Literatura Latino-Americana e do Caribe em Ciências da Saúde (LILACS), Medical Literature Analysis and Retrieval System Online (MEDLINE) via PubMed, Base de Dados de Enfermagem (BDENF) e Scientific Electronic Library Online (SciELO), utilizando os descritores: Palliative Care, Nursing e Primary Health Care. Resultados: Foram analisados 17 artigos após seleção sistemática, sintetizados em um quadro com seus principais resultados e agrupados em três categorias: capacitação em CP: uma barreira para atuaçáo do enfermeiro na APS; percepçóes, experiências e práticas dos enfermeiros nos CP; o papel do enfermeiro na equipe multiprofissional de CP. Conclusáo: Notou-se que os enfermeiros possuíam conhecimento superficial acerca dos CP na APS, evidenciando a necessidade de educaçáo continuada para promover a sua atuação em CP. Ademais, estudos com maior rigor metodológico com o foco no enfermeiro como agente disseminador da prática são necessários. Palavras-chave: cuidados paliativos; enfermeiras e enfermeiros; assistência centrada no paciente; atençáo primária à saúde; enfermagem domiciliar.

\section{ABSTRACT}

Introduction: The role of nursing professionals in palliative care (PC) in Primary Health Care (PHC) aims to promote the quality of life of individuals and their families as a guarantee of comprehensive humanized and dignified care, improving the way to cope with the disease and minimizing suffering. Objective: To analyze and synthesize the scientific production related to the nurse's assistance to the individual in PC in PHC. Method: Integrative literature review carried out in the databases of Latin American and Caribbean Health Sciences Literature (LILACS), Medical Literature Analysis and Retrieval System Online (MEDLINE) by PubMed, Nursing Database (BDENF) and Scientific Electronic Library Online (SciELO), using the following descriptors: Palliative Care, Nursing and Primary Health Care. Results: 17 articles were analyzed after a systematic selection summarized in a table with their main results and grouped into three categories: training in PC: a barrier for nurses to work in PHC; perceptions, experiences, and practices of nurses regarding $\mathrm{PC}$; the role of nurses in the multiprofessional PC team. Conclusion: It was noticed that nurses had superficial knowledge about PC in PHC, demonstrating the need for continuing education to promote their role in PC. Furthermore, thorough methodological studies targeted to the nurse as agent disseminating the practice are needed.

Key words: palliative care; nurses; patient-centered care; primary health care; home health nursing.
RESUMEN

Introducción: El rol de los profesionales de enfermería en los cuidados paliativos (CP) en la Atención Primaria de Salud (APS) tiene como objetivo promover la calidad de vida de las personas y sus familias como garantía de una atención integral, humanizada y digna, mejorando la forma de afrontar la enfermedad y minimizando sufrimiento. Objetivo: Analizar y sintetizar la producción científica relacionada con la asistencia del enfermero al individuo en CP en APS. Método: Revisión integradora de la literatura realizada en las bases de datos de Literatura Latinoamericana y del Caribe en Ciencias de la Salud (LILACS), Medical Literature Analysis and Retrieval Sistem Online (MEDLINE) a través de PubMed, Banco de Datos de Enfermería (BDENF) y Scientific Electronic Library Online (SciELO), utilizando los descriptores: Palliative Care, Nursing y Primary Health Care. Resultados: Se analizaron 17 artículos después de una selección sistemática, resumidos en una tabla con sus principales resultados y agrupados en tres categorías: capacitación en $\mathrm{CP}$ : barrera para el trabajo de enfermería en APS; percepciones, experiencias y prácticas de enfermeros en $\mathrm{CP}$; el papel de los enefermeros en el equipo multiprofesional de CP. Conclusión: Se observó que los enfermeros tenían conocimientos superficiales sobre CP en la APS, evidenciando la necesidad de una educación continua para promover su actuación en CP. Además, se necesitan estudios metodológicos más rigurosos, con foco en lo enfermero como agente divulgador de la práctica.

Palabras clave: cuidados paliativos; enfermeras y enfermeros; atención dirigida al paciente; atención primaria de salud; cuidados de enfermería en el hogar.

\footnotetext{
1-7Universidade Federal de Sergipe (UFS). Lagarto (SE), Brasil. E-mails: luan-fonseca@hotmail.com; becorreia97@gmail.com; hellen-oliver@hotmail.com; jackelinemelosilva@gmail.com; cleyton-121@hotmail.com; laiseluemmy.98@gmail.com; simonekameo@hotmail.com. Orcid iD: https://orcid.org/0000-0002-29817327; Orcid iD: https://orcid.org/0000-0002-1930-224X; Orcid iD: https://orcid.org/0000-0002-3435-032X; Orcid iD: https://orcid.org/0000-0003-4631-3646; Orcid iD: https://orcid.org/0000-0001-5616-7625; Orcid iD: https://orcid.org/0000-0002-8609-6108; Orcid iD: https://orcid.org/0000-0002-0035-2415
} Endereço para correspondência: Luan dos Santos Fonseca. Rua François Half, 655, apto 302 - Atalaia. Aracaju (SE), Brasil. CEP 49037-000. E-mail: Iuan-fonseca@hotmail.com 


\section{INTRODUÇÃO}

Nas últimas décadas, o Brasil vem passando por uma transiçáo demográfica a partir do aumento da expectativa de vida da população, que atualmente é de 76,6 anos conforme demonstrado pelo Instituto Brasileiro de Geografia e Estatística (IBGE) ${ }^{1}$. Atrelado ao maior número de idosos, há o aumento da incidência de doenças crônicas não transmissíveis, como neoplasias, problemas cardiovasculares e neurológicos. Nesse contexto, surge a necessidade de incorporar novas maneiras de lidar com pacientes que apresentem essas condiçóes, uma delas: os cuidados paliativos $(\mathrm{CP})^{1,2}$.

Os CP são definidos como cuidado holístico, o qual visa atender às necessidades de indivíduos com enfermidades que ameacem sua vida, fornecendo alívio de sintomas e melhorando a qualidade de vida destes, dos seus familiares e cuidadores. Nesse sentido, os CP favorecem o reconhecimento precoce de patologias que ameacem a vida, a partir da avaliaçáo cuidadosa e minuciosa da dor, controle de sintomas e de outras manifestaçóes físicas, sociais, psicológicas e espirituais ${ }^{3}$.

No Brasil, de acordo com a Academia Nacional de Cuidados Paliativos (ANCP) ${ }^{4}$, até o ano de 2018, existiam 177 serviços de CP. O início dessa prática no território brasileiro surgiu no ano 2000, apesar de ser abordada desde 1990. Por conseguinte, os CP podem ser ofertados a qualquer cliente, seja ele criança, adulto ou idoso, que possua doenças crônicas ou ameaçadoras da vida ${ }^{4}$.

Considerando a Atenção Primária à Saúde (APS) como o primeiro nível de atenção à saúde, caracterizada como conjunto de açôes com vistas à prevenção, promoçáo, tratamento, reabilitação e manutenção da saúde, com o objetivo de promover a atenção integral, constitui-se, portanto, uma ferramenta fundamental para o acompanhamento dos indivíduos em $\mathrm{CP}^{5}$.

Em países como Bélgica e França, os CP domiciliares são subsidiados por meio de programas de incentivos financeiros do Estado que custeiam não só o paciente, mas também os cuidadores, enquanto a Alemanha somente financia o paciente. Nesses três países, existem equipes multidisciplinares que atuam para fornecer conhecimento e apoio aos profissionais de saúde e cuidadores informais em CP, o acesso a esse serviço é gratuito nos países citados ${ }^{6}$.

Assim como nesses países, no Brasil, a função da APS não é ofertar cuidados do tipo Home Care, e sim dar suporte aos pacientes e à família, inclusive capacitando familiares ${ }^{7}$. Neste contexto, o profissional de enfermagem que atua em $\mathrm{CP}$ é visto pelo usuário como aquele que irá restabelecer sua saúde, reduzindo o sentimento de vulnerabilidade. Além disso, o enfermeiro é capaz de promover o controle de sintomas, realizar técnicas específicas como hipodermóclise, curativos, promover comunicação terapêutica, ofertar cuidados espirituais, realizar a manutenção da higiene, manter comunicação com a equipe multidisciplinar e prover medidas de conforto junto à família ${ }^{8-11}$.

Apesar da importância e da urgência em implementar os CP, há ainda resistência na implementação por razóes políticas e educacionais; nesse sentido, como meio de intervir em uma dessas lacunas - a educacional - com vistas a construir informaçóes sólidas como fomento ao desenvolvimento de prática clínica dos $\mathrm{CP}$, objetivou-se com o presente estudo: analisar e sintetizar a produção científica relacionada à assistência do enfermeiro na APS ao indivíduo em $\mathrm{CP}$.

\section{MÉTODO}

Trata-se de uma revisão integrativa da literatura que busca identificar, analisar e sintetizar pesquisas e conclusôes de fontes diversas em um tópico comum para viabilizar a incorporação de evidências na prática clínica ${ }^{12}$.

Esta revisão foi conduzida por meio de um estudo sistemático da literatura ${ }^{12}$ seguindo as etapas: (1) Elaboraçáo da questáo norteadora; (2) Busca na base de dados pelas literaturas; (3) Análise crítica dos estudos; (4) Seleção dos artigos; (5) Avaliação da qualidade metodológica; (6) Extração dos dados; (7) Síntese dos dados; (8) Avaliação da evidência dos estudos.

As etapas resumem-se em: identificação da questão da pesquisa, com a finalidade de esclarecer o objetivo do estudo e o resultado que pretende obter; identificação dos estudos, nessa etapa a busca abrangente na literatura foi realizada e a análise crítica foi implementada; seleção dos estudos, nesse passo uma equipe foi designada para abordar os estudos, selecioná-los e extrair os dados necessários; análise dos dados, os dados foram extraídos das fontes e as suas características foram analisadas, podendo-se aplicar categorias para facilitar esse processo ${ }^{13}$. Por fim, foram extraídos os dados por meio do uso adaptado de um instrumento, e síntese dos estudos, com a finalidade de elencar os principais resultados e pontos debatidos em cada artigo selecionado ${ }^{13}$. Essas etapas foram estudadas, estratificadas nos oito passos já citados e seguidas sistematicamente neste estudo.

Para atingir o objetivo, a seguinte questão norteadora foi elaborada: "Qual tem sido a atuação do enfermeiro da APS na assistência domiciliar a indivíduos em CP?".

A coleta de dados foi realizada no mês de julho de 2020. Para a seleção dos artigos, foram consultadas as bases de dados Literatura Latino-Americana e do Caribe em Ciências da Saúde (LILACS), Medical Literature Analysis and Retrieval System Online (MEDLINE) via PubMed, 
Base de Dados de Enfermagem (BDENF) e Scientific Electronic Library Online (SciELO), utilizando-se os Descritores em Ciências da Saúde (DeCS) e do Medical Subject Headings (MeSH) junto ao operador booleano "AND". Desse modo, a seguinte estratégia de busca foi utilizada nas bases selecionadas: "Palliative Care" (AND) "Nursing" (AND) "Primary Health Care".

Para a seleção das publicações, os critérios de inclusão aplicados foram publicaçóes disponíveis em quaisquer anos até o primeiro semestre de 2020 e artigos nos idiomas: inglês, português e espanhol. Foram excluídos os artigos não primários, como os de opinião, revisão, teses e monografias.

O processo de identificação, seleção e inclusão dos estudos primários ocorreu em três etapas. Na primeira etapa, foi efetuada a identificação dos estudos por meio dos descritores e aplicação dos filtros, totalizando 2.070 artigos (Figura 1). Na segunda, procedeu-se à leitura dos títulos e resumos dos artigos, à luz dos critérios de inclusão e exclusão, sendo selecionados 75 artigos. Após a leitura destes na íntegra, selecionaram-se 17 artigos. $\mathrm{O}$ estudo atendeu aos aspectos éticos, uma vez que os direitos autorais das pesquisas utilizadas foram respeitados conforme a Lei de Direitos Autorais ${ }^{14}$.

Para a coleta e análise dos dados, utilizou-se instrumento validado $^{15}$, o qual foi adaptado para atender ao objetivo do estudo. Diferentes informaçôes são exigidas pelo instrumento: identificação do estudo, instituição-sede do estudo, características metodológicas, rigor metodológico, entre outros. Os itens de interesse priorizados no instrumento foram: título, periódico, ano de publicaçáo, delineamento e resultados ${ }^{15}$. As mesmas variáveis utilizadas para a extração dos dados foram também para a disposição dos resultados. A análise de conteúdo foi empregada para a categorização dos achados seguindo as etapas de pré-análise, regra de exaustividade, exploração do material e tratamento dos dados, inferências e interpretaçôes, conforme preconizado por Bardin ${ }^{16}$. Como produto da análise, foram obtidas três categorias: Capacitação em CP: uma barreira para a atuação do enfermeiro na APS; Percepçóes, experiências e prática dos enfermeiros nos CP; $\mathrm{O}$ papel do enfermeiro na equipe multiprofissional de CP.

A descrição da busca nas bases de dados está apresentada na Figura 1.

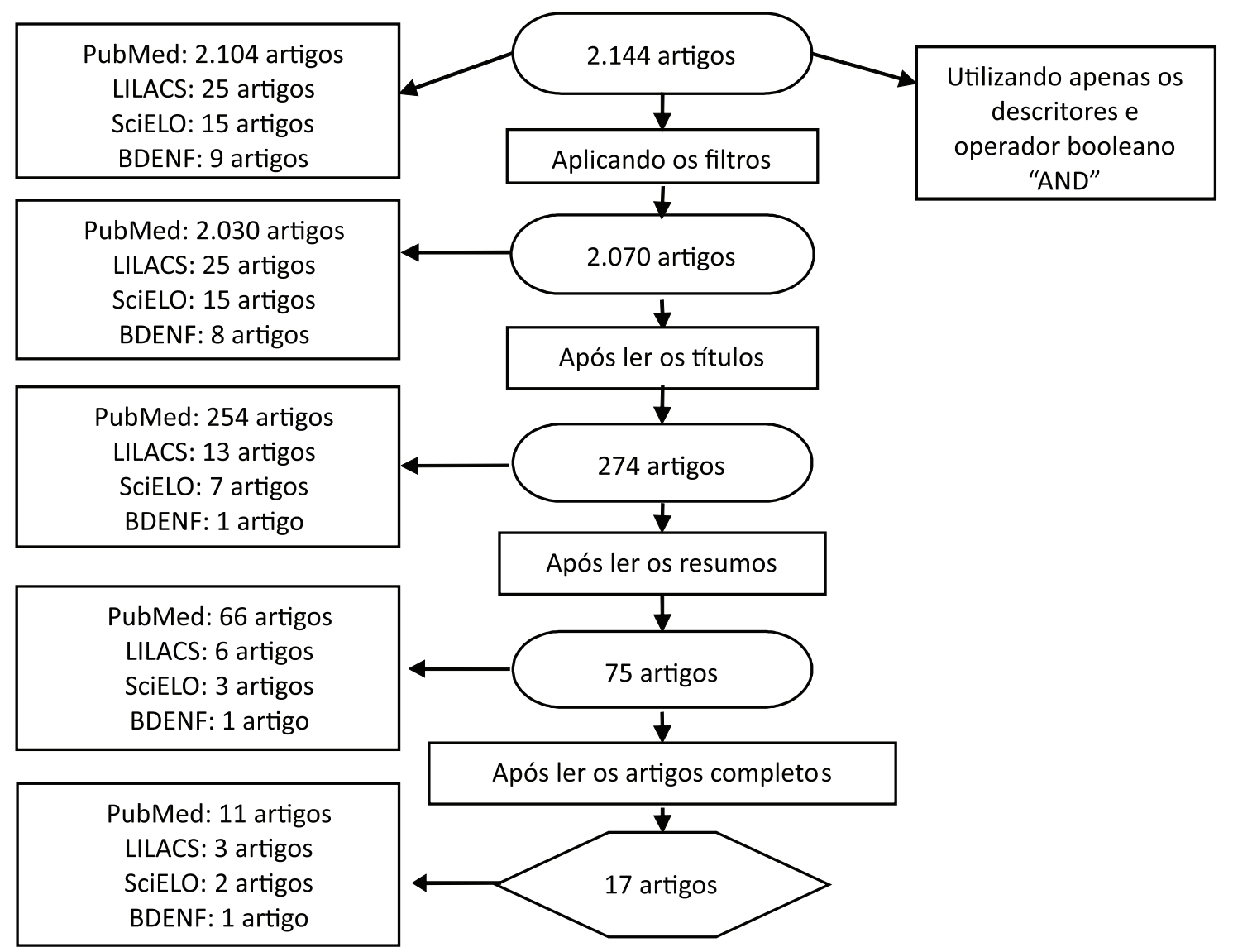

Figura 1. Estratégias de busca para inclusão dos artigos 


\section{RESULTADOS}

A amostra final consistiu de 17 artigos. A maioria foi publicada no ano de 2018, correspondendo a seis artigos, sendo os demais publicados em 2012 (1), 2015 (3), 2016 (2), 2017 (2), 2019 (2), 2020 (1). O idioma predominante dos manuscritos foi a língua inglesa (12 artigos); em seguida, a língua portuguesa (3); e língua espanhola (2), conforme o Quadro 1.

Os resultados indicam que é recente a ascendência no número de artigos sobre a assistência ao paciente em CP na APS, concentrando-se em sua maioria nos anos de 2015 a 2018; além disso o número de pesquisas sobre o assunto cresce a cada ano em decorrência do grande aumento das taxas de mortalidade ${ }^{17}$.

Quadro 1. Distribuição das referências de acordo com periódico, título, delineamento, ano de publicação e resultados

\begin{tabular}{|c|c|c|c|c|}
\hline Autor/Ano & Periódico & Tífulo & Delineamento & Resultados \\
\hline $\begin{array}{l}\text { Sousa e } \\
\text { Alves, } 2015^{18}\end{array}$ & $\begin{array}{l}\text { Acta Paul } \\
\text { Enferm }\end{array}$ & $\begin{array}{l}\text { Nursing competencies } \\
\text { for palliative care in } \\
\text { home care }\end{array}$ & $\begin{array}{l}\text { Descritivo } \\
\text { exploratório, } \\
\text { quantitativo }\end{array}$ & $\begin{array}{l}\text { Os enfermeiros demonstraram } \\
\text { consenso entre as competências } \\
\text { gerais e específicas para o } \\
\text { desenvolvimento do CP em } \\
\text { domicílio por meio da assistência } \\
\text { multidisciplinar e transdisciplinar }\end{array}$ \\
\hline $\begin{array}{l}\text { Pesut et al., } \\
2015^{19}\end{array}$ & $\begin{array}{l}\text { Rural Remote } \\
\text { Health }\end{array}$ & $\begin{array}{l}\text { Feasibility of a rural } \\
\text { palliative supportive } \\
\text { service }\end{array}$ & $\begin{array}{l}\text { Delineamento } \\
\text { não informado }\end{array}$ & $\begin{array}{l}\text { Para viabilizar a implantação dos } \\
\text { CP na comunidade rural, a equipe } \\
\text { de enfermagem realizou divulgação } \\
\text { do serviço, recrutamento e visitas } \\
\text { quinzenais aos pacientes }\end{array}$ \\
\hline $\begin{array}{l}\text { Dahlin et al., } \\
2016^{20}\end{array}$ & J Palliat Med & $\begin{array}{l}\text { The advanced } \\
\text { practice registered } \\
\text { nurses palliative care } \\
\text { externship: a model } \\
\text { for primary palliative } \\
\text { care education }\end{array}$ & $\begin{array}{l}\text { Descritivo de } \\
\text { coorte }\end{array}$ & $\begin{array}{l}\text { Inicialmente, os enfermeiros } \\
\text { afirmaram ter pouco ou nenhum } \\
\text { conhecimento sobre CP. Após o } \\
\text { curso de educação continuada, } \\
\text { demonstraram maior conhecimento e } \\
\text { confiança no desenvolvimento dos CP }\end{array}$ \\
\hline $\begin{array}{l}\text { Pereira et al., } \\
2017^{21}\end{array}$ & $\begin{array}{l}\text { Rev Enferm } \\
\text { UFPE on line }\end{array}$ & $\begin{array}{l}\text { Significados dos } \\
\text { cuidados paliativos na } \\
\text { ótica de enfermeiros } \\
\text { e gestores da Atenção } \\
\text { Primária à Saúde }\end{array}$ & $\begin{array}{l}\text { Descritivo de } \\
\text { abordagem } \\
\text { qualitativa }\end{array}$ & $\begin{array}{l}\text { Os profissionais de enfermagem } \\
\text { demonstram que entendem a } \\
\text { importância do CP, no entanto, } \\
\text { relatam que há um déficit de } \\
\text { aprendizado na equipe e que isso } \\
\text { interfere na assistência }\end{array}$ \\
\hline $\begin{array}{l}\text { Gálvez } \\
\text { Ramírez et } \\
\text { al., } 2017^{22}\end{array}$ & $\begin{array}{l}\text { Rev Eugenio } \\
\text { Espejo }\end{array}$ & $\begin{array}{l}\text { Caso relacionado con } \\
\text { el proceso asistencial } \\
\text { integrado cuidados } \\
\text { paliativos en atención } \\
\text { primaria de salud }\end{array}$ & Relato de Caso & $\begin{array}{l}\text { Expõe sobre a aplicabilidade da } \\
\text { sistematização da assistência de } \\
\text { enfermagem na prática dos CP }\end{array}$ \\
\hline $\begin{array}{l}\text { Nakazawa et } \\
\text { al., } 2018^{23}\end{array}$ & $\begin{array}{l}\text { J Pain } \\
\text { Symptom } \\
\text { Manage }\end{array}$ & $\begin{array}{l}\text { Changes in nurses' } \\
\text { knowledge, difficulties, } \\
\text { and self-reported } \\
\text { practices toward } \\
\text { palliative care for } \\
\text { cancer patients in } \\
\text { Japan: an analysis } \\
\text { of two nationwide } \\
\text { representative surveys } \\
\text { in } 2008 \text { and } 2015\end{array}$ & Estudo de coorte & $\begin{array}{l}\text { Após a aplicação de escalas de } \\
\text { teste de conhecimento, foi possível } \\
\text { verificar melhorias significativas } \\
\text { no desempenho das práticas } \\
\text { recomendadas em CP, nos } \\
\text { enfermeiros com maior tempo de } \\
\text { treinamento }\end{array}$ \\
\hline $\begin{array}{l}\text { Johansen e } \\
\text { Ervik, 2018 }\end{array}$ & $\begin{array}{l}\text { BMC Health } \\
\text { Serv Res }\end{array}$ & $\begin{array}{l}\text { Teamwork in primary } \\
\text { palliative care: general } \\
\text { practitioners' and } \\
\text { specialised oncology } \\
\text { nurses' complementary } \\
\text { competencies }\end{array}$ & $\begin{array}{l}\text { Descritivo } \\
\text { qualitativo }\end{array}$ & $\begin{array}{l}\text { Com base nas competências de } \\
\text { uma enfermeira especializada em } \\
\text { CP primários, o estudo aponta sua } \\
\text { capacidade de descrever e observar } \\
\text { com precisão sinais e sintomas, } \\
\text { valendo-se de sua experiência e } \\
\text { conhecimento atualizado em CP na } \\
\text { APS }\end{array}$ \\
\hline
\end{tabular}


Quadro 1. continuação

\begin{tabular}{|c|c|c|c|c|}
\hline Autor/Ano & Periódico & Título & Delineamento & Resultados \\
\hline $\begin{array}{l}\text { Seow et al., } \\
2018^{25}\end{array}$ & $\begin{array}{l}\text { BMC Palliat } \\
\text { Care }\end{array}$ & $\begin{array}{l}\text { Benchmarking time to } \\
\text { initiation of end-of-life } \\
\text { homecare nursing: } \\
\text { a population-based } \\
\text { cancer cohort study in } \\
\text { regions across Canada }\end{array}$ & $\begin{array}{l}\text { Coorte } \\
\text { retrospectivo }\end{array}$ & $\begin{array}{l}\text { Há uma variação para o tempo } \\
\text { de início da assistência domiciliar, } \\
\text { todavia, a enfermagem em CP } \\
\text { representou a opção majoritária } \\
\text { favorecendo mais dias de vida ao } \\
\text { paciente }\end{array}$ \\
\hline $\begin{array}{l}\text { Mkwinda e } \\
\text { Lekalakala- } \\
\text {-Mokgele, } \\
2016^{26}\end{array}$ & Curationis & $\begin{array}{l}\text { Palliative care needs in } \\
\text { Malawi: care received } \\
\text { by people living with } \\
\text { HIV }\end{array}$ & $\begin{array}{l}\text { Descritivo e } \\
\text { exploratório, } \\
\text { com abordagem } \\
\text { qualitativa }\end{array}$ & $\begin{array}{l}\text { Os resultados evidenciaram a } \\
\text { necessidade de os enfermeiros } \\
\text { conhecerem as principais demandas } \\
\text { para o cuidado da pessoa que vive } \\
\text { com HIV }\end{array}$ \\
\hline $\begin{array}{l}\text { Mancilla et } \\
\text { al., } 2018^{27}\end{array}$ & $\begin{array}{l}\text { Anales del } \\
\text { Sis San } \\
\text { Navarra }\end{array}$ & $\begin{array}{l}\text { Percepciones de los } \\
\text { profesionales sobre } \\
\text { la atención prestada, } \\
\text { obstáculos y dilemas } \\
\text { éticos relacionados } \\
\text { con el final de la vida } \\
\text { en hospitales, centros } \\
\text { de Atención Primaria } \\
\text { y residencias de } \\
\text { ancianos }\end{array}$ & $\begin{array}{l}\text { Descritivo, } \\
\text { transversal e } \\
\text { multicêntrico }\end{array}$ & $\begin{array}{l}\text { Os profissionais da APS não } \\
\text { encontraram obstáculos para } \\
\text { desenvolver o cuidado, todavia, para } \\
\text { a produção do cuidar no asilo, houve } \\
\text { dilemas éticos que se tornaram } \\
\text { empecilhos }\end{array}$ \\
\hline $\begin{array}{l}\text { Silva et al., } \\
2018^{28}\end{array}$ & Rev APS & $\begin{array}{l}\text { Construindo a linha } \\
\text { de cuidado do } \\
\text { paciente oncológico } \\
\text { paliativo em um } \\
\text { município do sul } \\
\text { do Brasil: relato de } \\
\text { experiência }\end{array}$ & Relato descritivo & $\begin{array}{l}\text { Evidenciou-se, a partir de uma } \\
\text { intervenção, que existem lacunas } \\
\text { no ensino dos profissionais, estes } \\
\text { relataram dificuldade com pacientes } \\
\text { oncológicos em CP. Constatou-se } \\
\text { a necessidade ações de educação } \\
\text { permanente para a construção da } \\
\text { linha de cuidado integral }\end{array}$ \\
\hline $\begin{array}{l}\text { Klop et al., } \\
2020^{29}\end{array}$ & $\begin{array}{l}\text { BMC Palliat } \\
\text { Care }\end{array}$ & $\begin{array}{l}\text { Strengthening the } \\
\text { spiritual domain in } \\
\text { palliative care through } \\
\text { a listening consultation } \\
\text { service by spiritual } \\
\text { caregivers in Dutch } \\
\text { PaTz-groups: an } \\
\text { evaluation study }\end{array}$ & $\begin{array}{l}\text { Descritivo } \\
\text { qualitativo }\end{array}$ & $\begin{array}{l}\text { Abordam o não cumprimento de } \\
\text { uma das dimensões da saúde } \\
\text { no processo de cuidado, a } \\
\text { espiritualidade }\end{array}$ \\
\hline $\begin{array}{l}\text { Sijabat et al., } \\
2019^{30}\end{array}$ & Enf Clinica & $\begin{array}{l}\text { Experiences of } \\
\text { palliative care nurses } \\
\text { in providing home- } \\
\text { based care for patient } \\
\text { with advanced cancer }\end{array}$ & $\begin{array}{l}\text { Fenomenológico } \\
\text { descritivo }\end{array}$ & $\begin{array}{l}\text { Os enfermeiros assumiram o foco } \\
\text { biomédico, atentando-se mais ao } \\
\text { alívio dos sintomas como dor, falta } \\
\text { de ar e vômitos, desconsiderando o } \\
\text { cuidado holístico }\end{array}$ \\
\hline $\begin{array}{l}\text { Chong e } \\
\text { Abdullah, } \\
2017^{31}\end{array}$ & $\begin{array}{l}\text { Am J Hosp } \\
\text { Palliat Med }\end{array}$ & $\begin{array}{l}\text { Community palliative } \\
\text { care nurses' } \\
\text { challenges and coping } \\
\text { strategies on delivering } \\
\text { home-based pediatric } \\
\text { palliative care: a } \\
\text { qualitative study }\end{array}$ & Qualitativo & $\begin{array}{l}\text { Evidencia a abordagem } \\
\text { interdisciplinar colaborativa para a } \\
\text { prática de CP pediátricos }\end{array}$ \\
\hline $\begin{array}{l}\text { Danielsen et } \\
\text { al., } 2018^{32}\end{array}$ & $\begin{array}{l}\text { BMC Palliat } \\
\text { Care }\end{array}$ & $\begin{array}{l}\text { Experiences and } \\
\text { challenges of home } \\
\text { care nurses and } \\
\text { general practitioners in } \\
\text { home-based palliative } \\
\text { care - a qualitative } \\
\text { study }\end{array}$ & $\begin{array}{l}\text { Qualitativo, } \\
\text { com dimensão } \\
\text { fenomenológica }\end{array}$ & $\begin{array}{l}\text { As enfermeiras enfatizaram a } \\
\text { importância de uma relação } \\
\text { confiável criada a partir de empatia } \\
\text { e tempo para ouvir paciente e } \\
\text { familiar. Além disso, ressaltaram a } \\
\text { necessidade de planejamento para } \\
\text { os CP no domicílio }\end{array}$ \\
\hline
\end{tabular}


Quadro 1. continuação

\begin{tabular}{|l|l|l|l|l|}
\hline Autor/Ano & Periódico & \multicolumn{1}{|c|}{ Título } & Delineamento & \multicolumn{1}{c|}{ Resultados } \\
\hline $\begin{array}{l}\text { Gágyor et al., } \\
2019^{33}\end{array}$ & Fam Pract & $\begin{array}{l}\text { Ethical challenges in } \\
\text { primary care: a focus } \\
\text { group study with } \\
\text { general practitioners, } \\
\text { nurses and informal } \\
\text { caregivers }\end{array}$ & Qualitativo & $\begin{array}{l}\text { Para os enfermeiros, os requisitos } \\
\text { burocráticos, os conflitos de } \\
\text { interesses financeiros entre os } \\
\text { familiares e pacientes, e a incerteza } \\
\text { sobre o que fazer com os usuários } \\
\text { eram barreiras para prestação de } \\
\text { cuidado de alta qualidade em CP }\end{array}$ \\
\hline $\begin{array}{l}\text { Yamagishi et } \\
\text { al., 2012 }\end{array}$ & $\begin{array}{l}\text { J Pain and } \\
\text { Symptom } \\
\text { Manage }\end{array}$ & $\begin{array}{l}\text { Providing palliative } \\
\text { care for cancer } \\
\text { patients: the views } \\
\text { and exposure of } \\
\text { community general } \\
\text { practitioners and } \\
\text { district nurses in Japan }\end{array}$ & Transversal & $\begin{array}{l}\text { O estudo apontou que 93\% dos } \\
\text { serviços de enfermagem distrital } \\
\text { estavam dispostos a utilizar consulta } \\
\text { de CP e tinham domínio sobre uso } \\
\text { de opioides no serviço prestado }\end{array}$ \\
\hline
\end{tabular}

Legendas: $\mathrm{CP}$ = Cuidados paliativos; APS = Atenção Primária à Saúde; HIV = Vírus da imunodeficiência humana.

\section{DISCUSSÃO}

\section{CAPACITAÇÃO EM CP: UMA BARREIRA PARA A ATUAÇÃO DO ENFERMEIRO NA APS}

Um estudo ${ }^{23}$ descreveu a utilização das seguintes ferramentas de avaliação do conhecimento, e dificuldades em relação aos CP, de 2008 a 2015, no Japão: Palliative Care Knowledge (PCKT), Palliative Care Difficulty Scale (PCDS) e Palliative Care Self-Reported Practices Scale (PCPS). Foram identificadas melhoria no nível de conhecimento e diminuição do número de dificuldades associadas à prestação de $\mathrm{CP}$, em virtude dos programas de educação em enfermagem. Além disso, verificou-se a necessidade de enfermeiros especialistas nas equipes de $\mathrm{CP}$, bem como a inclusão de $\mathrm{CP}$ como um elemento obrigatório e regular de educação para os profissionais de saúde ${ }^{23}$.

Identificou-se nos estudos ${ }^{20,21,28}$ que, durante a graduação do curso de enfermagem, existe a falta de debate sobre os CP na grade curricular ou que são abordados de maneira bastante superficial. Nesse sentido, em uma pesquisa com gestores da APS, verificou-se que as equipes de Estratégia Saúde da Família desconhecem o significado dos CP, além disso foi relatado que a formação acadêmica dos profissionais é superficial acerca da temática ${ }^{21}$. Essa informação confirma o que propóe a ANCP - que a maioria das equipes trabalha sem educação formal e o conhecimento baseia-se nas iniciativas autodidáticas ${ }^{8}$. Por isso, a importância da realização de educação continuada, a fim de superar essas lacunas, bem como de alguns sentimentos por parte dos profissionais, como o de impotência, o medo e a insegurança para lidar com a finitude ${ }^{28}$. Segundo Dahlin et al. ${ }^{20}$, o conhecimento e a autoconfiança dos profissionais melhoraram após cursos de educação continuada sobre CP.
A realidade da formação em enfermagem dentro do âmbito das instituições de ensino não flexibiliza a potencialidade do aluno para a atuaçáo profissional em diversos contextos ${ }^{35}$. Desse modo, a formaçáo generalista é uma realidade encontrada e configura-se como uma das dificuldades para a atuação da equipe de enfermagem em $\mathrm{CP}$, sendo necessária a capacitação para a reversão do quadro ${ }^{35}$. A oferta de capacitação por intermédio de práticas ativas, como a simulação realística, constitui um importante método para explanação de aspectos objetivos e subjetivos que norteiam a atençáo em $\mathrm{CP}$, além de preparar a atuação do graduando, profissional ou residente para a prática encontrada na realidade de trabalho e nortear o desenvolvimento de habilidades, como a escuta ativa, empatia e comunicação ${ }^{36}$.

\section{PerCePÇÕES, EXPERIÊNCIAS E PRÁtICA dOS ENFERMEIROS NOS CP}

O enfermeiro da comunidade está no primeiro nível de acesso aos serviços de saúde, obtendo, assim, mais contato com a população, fornecendo um cuidado mais preciso às demandas do paciente ${ }^{37}$. Nesse sentido, um estudo de Gálvez Ramírez et al. ${ }^{22}$ discute sobre a importância da atuação do enfermeiro na APS aos pacientes em CP, além da família e comunidade, sendo esta a essência na promoção de qualidade de vida. O desempenho da função deve dar-se por meio da capacidade de personalizar o atendimento, boa comunicaçáo com a família e paciente, continuidade dos cuidados e capacidade de apoiar o papel do cuidador familiar ${ }^{22}$.

No mesmo estudo, para a realização de avaliaçáo dos familiares, cuidador e do paciente, foram utilizados os seguintes instrumentos: Zarit (avalia a sobrecarga do cuidador), Apgar familiar (identificação do suporte familiar) e o modelo das 14 necessidades básicas de Virgínia 
Henderson ${ }^{22}$. Posteriormente, para implementação e desenvolvimento do plano de cuidado, foram utilizadas a North American Nursing Diagnosis Associativo (NANDA); a Nursing Outcomes Classification (NOC); e a Nursing Interventions Classification (NIC) ${ }^{22}$. A partir da utilização dessas taxonomias, implementaram-se os potenciais diagnósticos prioritários, metas e intervençōes. Obtiveram-se como diagnósticos: Ansiedade antes da morte e risco de fadiga do papel do cuidador; Metas: alcance da pontuação máxima (sempre demonstrada) nos indicadores expressão de desejo de viver e expressão de motivos de viver, alcance da pontuação máxima (nenhum) para os indicadores medo e frustração; Intervençôes: apoio emocional ao paciente por meio das seguintes atividades (abraçar ou tocar o paciente para fornecer suporte; encorajar o paciente para expressar os sentimentos de ansiedade, raiva e tristeza; proporcionar apoio durante as fases do luto e ajuda na tomada de decisóes); e apoio ao cuidador principal por meio dessas intervençôes (determinar nível de conhecimento do cuidador; apoiar as tomadas de decisóes e dificuldades enfrentadas pelo cuidador etc. $)^{22}$.

Em outra pesquisa, com o público de pacientes adolescentes com neoplasia em CP, também foram implementadas as taxonomias NANDA, NIC, NOC, tendo como referencial teórico o Modelo de Adaptaçáo de Callista Roy, a fim de assegurar uma assistência de enfermagem baseada em evidência, com a finalidade de ofertar cuidado integral e humanizado ${ }^{38}$.

Em um estudo com os enfermeiros oncológicos e paliativistas, observou-se que estes contribuíam no cuidado dos pacientes com habilidades e competências, como observar e descrever com precisáo sinais e sintomas do paciente, com base nos seus conhecimentos e em experiências anteriores com CP. Além disso, por possuírem conhecimento em CP, também sugeriam possível conduta terapêutica em relação à analgesia ${ }^{24}$.

Em outra análise, foi observada a capacidade dos enfermeiros em antecipar e responder às necessidades humanas em $\mathrm{CP}$, bem como compreender suas próprias limitações na busca de ajuda referente às práticas complexas ${ }^{18}$. Nesse sentido, relataram problemas relacionados à falta de confiança, a problemas entre as famílias relacionados a conflitos financeiros, a problemas relacionados ao conhecimento e autonomia do enfermeiro na prestação do cuidado e a conflitos entre a equipe multidisciplinar, principalmente entre o médico da família e demais profissionais ${ }^{33}$.

Foi possível identificar que ainda há um grande desafio na implementação dos CP na APS, visto que ainda existem falhas no conhecimento dos profissionais. Em outro estudo, enfermeiros associavam os $\mathrm{CP}$ ao tempo de sobrevida do paciente, e verificaram que os familiares não recebiam assistência. Além disso, os enfermeiros relacionaram os CP a uma terapêutica alternativa ou ainda inadequada, além de considerarem apenas pacientes oncológicos elegíveis para a prestação de cuidados ${ }^{25}$. Nesse sentido, os princípios dos CP redigidos pela Organização Mundial da Saúde (OMS), como fornecer uma avaliação detalhada por profissionais capacitados, oferecer sistema de suporte de apoio, e integrar os aspectos psicológicos e espirituais na assistência, podem subsidiar a efetivação dos $\mathrm{CP}^{8}$.

\section{PAPEL DO ENFERMEIRO NA EQUIPE MULTIPROFISSIONAL DE CP}

$A$ base da equipe interdisciplinar deve ser composta por assistente social, enfermeiro, médico e psicólogo. Podendo ser integrada ainda por: educadores, fisioterapeutas, técnicos de enfermagem, fonoaudiólogos, musicoterapeutas, nutricionistas, psiquiatra, terapeutas ocupacionais e um conselheiro espiritual ou capeláo, para poder ofertar uma assistência integral. A quantidade de especialidades varia conforme disponibilidade de recursos ${ }^{39,40}$.

Dois estudos abordaram a composição da equipe prestadora de $\mathrm{CP}$, sendo uma composta por médico, enfermeiro, fisioterapeuta e terapeuta ocupacional, e a outra somente com enfermeiros e médicos ${ }^{19,24}$. Em virtude disso, o paciente que necessitaria de um acompanhamento com profissionais de diversas especialidades pode não ter todos os sintomas amenizados, bem como problemas espirituais e sociais náo corrigidos ou acompanhados corretamente por conta da estrutura da equipe assistencial ${ }^{39}$.

Portanto, fica claro que o cumprimento do papel de cada um dos profissionais que fazem parte desse processo é imprescindível, porém, nos mais diversos cenários que incluíam pacientes em $\mathrm{CP}$, os enfermeiros relatavam "sofrimento moral" e sentiam-se ignorados em decorrência das relaçóes hierárquicas; mesmo apresentando vasta experiência e tendo maior contato com os pacientes, suas indicaçóes e sugestóes não eram avaliadas para contribuir com a conduta terapêutica ${ }^{41}$. Nesse sentido, a comunicação é evidenciada como ferramenta fundamental no processo de integrar a assistência e na resolução de possíveis conflitos existentes entre as equipes ${ }^{20,42,43}$.

Diante do exposto, percebe-se, então, que tais condutas impactam diretamente na qualidade da assistência prestada pela equipe multiprofissional, e inviabilizavam a prática das diretrizes preconizadas em $\mathrm{CP}^{8}$. Assim, torna-se necessário que os gestores de serviços de saúde observem as vulnerabilidades dos profissionais para que sejam capazes de intervir, a fim de melhorar a qualidade do serviço prestado por meio de evidências, ofertar assistência humanizada, bem como melhorar a relação interpessoal dos profissionais para que se alcance a integralidade do cuidado nessa perspectiva ${ }^{11,39}$. 
A ausência de padronização na prática dos $\mathrm{CP}$, descrita nos estudos observados, foi um dos fatores que dificultaram a síntese dos resultados após a análise categórica, no entanto, proporcionou a ampla visualizaçáo da atuaçáo do enfermeiro na APS. Por isso, o estudo viabiliza que sejam desenvolvidas novas pesquisas que proporcionem o maior envolvimento dos profissionais de enfermagem com os $\mathrm{CP}$ na APS, visto que o conhecimento sobre o assunto é limitado e os pacientes necessitam de cuidado integral.

\section{CONCLUSÃO}

$\mathrm{Na}$ APS, os enfermeiros contribuem no cuidado dos pacientes com habilidades técnicas predominantemente gerais e competências relacionais, como observar e descrever com precisão sinais e sintomas e estabelecendo boa comunicação com a família e o paciente. Atuam apresentando lacunas, como a falta de aprimoramento, para desenvolver CP. Conforme apontado neste estudo, o conhecimento superficial dos profissionais sobre a temática dos CP e a falta de capacitaçóes configuram-se como principais barreiras a serem superadas para o avanço nessa área.

\section{CONTRIBUIÇÕES}

Todos os autores contribuíram substancialmente na concepção e/ou planejamento do estudo; na obtenção, análise e/ou interpretação dos dados; na redação e revisão crítica; e aprovaram a versão final a ser publicada.

\section{DECLARAÇÃO DE CONFLITO DE INTERESSES}

Nada a declarar.

\section{FONTES DE FINANCIAMENTO}

Não há.

\section{REFERÊNCIAS}

1. Instituto Brasileiro de Geografia e Estatística. Tábua completa de mortalidade para o Brasil - 2019: breve análise da evolução da mortalidade no Brasil [Internet]. Rio de Janeiro: IBGE; 2019 [acesso 2021 maio 20]. Disponível em: https://biblioteca.ibge.gov.br/ visualizacao/periodicos/3097/tcmb_2019.pdf

2. Souza MFM, Malta DC, França EB. Changes in health and disease in Brazil and its States in the 30 years since the Unified Healthcare System (SUS) was created. Cenk Saude Colet. 2018;23(6):1737-50. doi: https://doi. org/10.1590/1413-81232018236.04822018
3. World Health Organization. National cancer control programmes: policies and managerial guidelines [Internet]. 2nd ed. Geneva: WHO; 2002 [cited 2020 Dec 16]. Available from: https://apps.who.int/iris/bitstream/ handle/10665/42494/9241545577.pdf;jsessionid=06B1 0A6EFFECB17956F58F91007D9690? sequence $=1$

4. Academia Nacional de Cuidados Paliativos. Panorama de cuidados paliativos no Brasil [Internet]. Sáo Paulo: ANCP; 2018 [acesso 2021 maio 19]. Disponível em: https://paliativo.org.br/wp-content/uploads/2018/10/ Panorama-dos-Cuidados-Paliativos-no-Brasil-2018.pdf

5. Starfield B. Atenção primária: equilíbrio entre necessidades de saúde, serviços e tecnologia. Brasília: UNESCO, Ministério da Saúde; 2002.

6. Maetens A, Beernaert K, Deliens L, et al. Policy measures to support palliative care at home: a cross-country case comparison in three European Countries. J Pain Symptom Manage. 2017;54(4):523-9. doi: https://doi. org/10.1016/j.jpainsymman.2017.07.022

7. Queiroz TA, Ribeiro ACM, Guedes MVC, et al. Palliative care to the elderly in intensive care: the perspective of the nursing team. Texto Contexto Enferm. 2018;27(1):e1420016. doi: https://doi. org/10.1590/0104-07072018001420016

8. Academia Nacional de Cuidados Paliativos. Manual de cuidados paliativos ANCP [Internet]. 2. ed. ampl. atual. Rio de Janeiro: ANCP; 2012 [acesso $2020 \mathrm{dez}$ 16]. Disponível em: http://biblioteca.cofen.gov.br/ wp-content/uploads/2017/05/Manual-de-cuidadospaliativos-ANCP.pdf

9. Economist Intelligence Unit. The 2015 quality of death Index: ranking palliative care across the world. London, UK: EIU; 2015.

10. Andrade FLM, Sousa e Silva ME, Macêdo EL, et al. Dor oncológica: manejo clínico realizado por enfermeiros. Rev Univ Vale Rio Verde. 2018;8(1):3-16.

11. Lindolpho MC, Caldas CP, Sá SPC, et al. Cuidados de enfermagem ao idoso no fim da vida. Cien Cuid Saude. 2016;15(2):383-9. doi: https://doi.org/10.4025/ ciencuidsaude.v15i2.23904

12. Souza MT, Silva MD, Carvalho R. Integrative review: what is it? How to do it? Einstein (Sáo Paulo). 2010;8(1):102-6. doi: https://doi.org/10.1590/S1679-45082010RW1134

13. Whittemore R, Chao A, Jang M, et al. Methods for knowledge synthesis: an overview. Heart Lung. 2014;43(5):453-61. doi: https://doi.org/10.1016/j. hrtlng.2014.05.014

14. Presidência da República (BR). Lei no 12.853 , de 14 de agosto de 2013. Altera os arts. 5o , 68, 97, 98, 99 e 100 , acrescenta arts. 98-A, 98-B, 98-C, 99-A,99-B, 100A, 100-B e 109-A e revoga o art. 94 da Lei no 9.610, de 19 de fevereiro de1998, para dispor sobre a gestáo coletiva de direitos autorais, e dá outras providências. Diário Oficial da União. 2013 ago 15; Edição 157, Seção 1:1. 
15. Ursi ES, Gavão CM. Prevenção de lesões de pele no perioperatório: revisão integrativa da literatura. Rev Latino-Am Enfermagem. 2006;14(1):124-31. doi: https://doi.org/10.1590/S0104-11692006000100017

16. Santos FM. Análise de conteúdo: a visão de Laurence Bardin [resenha]. Rev Eletrônica Educ. 2012;6(1):383-7.

17. Sung H, Ferlay J, Siegel RL, et al. Global cancer statistics 2020: GLOBOCAN estimates of incidence and mortality worldwide for 36 cancers in 185 countries. CA Cancer J Clin. 2021;71(3):209-49. doi: https://doi.org/10.3322/ caac. 21660

18. Sousa JM, Alves ED. Nursing competencies for palliative care in home care. Acta Paul Enferm. 2015;28(3):4-6. doi: https://doi.org/10.1590/1982-0194201500044

19. Pesut B, Hooper BP, Robinson C, et al. Feasibility of a rural palliative supportive service. Rural Remote Health. 2015;15(2):3116. doi: https://doi.org/10.22605/ RRH3116

20. Dahlin C, Coyne PJ, Cassel JB. The advanced practice registered nurses palliative care externship: a model for primary palliative care education. J Palliat Med. 2016;19(7):753-9. doi: https://doi.org/10.1089/ jpm.2015.0491

21. Pereira DG, Fernandes J, Ferreira LS, et al. Significados dos cuidados paliativos na ótica de enfermeiros e gestores da atenção primária à saúde. Rev Enferm UFPE on line. 2017;11(Supl. 3):1357-64.

22. Gálvez Ramírez B, Samaniego Ruiz M, Cambil Martín J. Caso relacionado con el proceso asistencial integrado cuidados paliativos en atención primaria de salud. Rev Eugenio Espejo. 2017;11(2):65-74. doi: https://doi. org/10.37135/ee.004.03.08

23. Nakazawa $Y$, Kato M, Miyashita M, et al. Changes in nurses' knowledge, difficulties, and self-reported practices toward palliative care for cancer patients in Japan: an analysis of two nationwide representative surveys in 2008 and 2015. J Pain Symptom Manage. 2018;55(2):402-12. doi: https://doi.org/10.1016/j. jpainsymman.2017.08.034

24. Johansen ML, Ervik B. Teamwork in primary palliative care: general practitioners' and specialised oncology nurses' complementary competencies. BMC Health Serv Res. 2018;18(1):159. doi: https://doi.org/10.1186/ s12913-018-2955-7

25. Seow H, Qureshi D, Barbera L, et al. Benchmarking time to initiation of end-of-life homecare nursing: a population-based cancer cohort study in regions across Canada. BMC Palliat Care. 2018;17:70. doi: https://doi. org/10.1186/s12904-018-0321-5

26. Mkwinda E, Lekalakala-Mokgele E. Palliative care needs in Malawi: care received by people living with HIV. Curationis. 2016;39(1):1664. doi: https://doi. org/10.4102/curationis.v39i1.1664
27. Mancilla PG, Montoya-Juarez R, Marti-Garcia C, et al. Percepciones de los profesionales sobre la atención prestada, obstáculos y dilemas éticos relacionados con el final de la vida en hospitales, centros de Atención Primaria y residencias de ancianos. Anales Sis San Navarra. 2018;41(1):35-46. doi: https://doi. org/10.23938/ASSN.0170

28. Silva KF, Pucci VR, Flores TG, et al. Construindo a linha de cuidado do paciente oncológico paliativo em um município do sul do Brasil: relato de experiência. Rev APS. 2018;21(3):470-7.

29. Klop HT, Koper I, Schweitzer BPM, et al. Strengthening the spiritual domain in palliative care through a listening consultation service by spiritual caregivers in Dutch PaTz-groups: an evaluation study. BMC Palliat Care. 2020;19:92. doi: https://doi.org/10.1186/s12904-02000595-0

30. Sijabat M, Dahlia D, Waluyo A. Experiences of palliative care nurses in providing home-based care for patient with advanced cancer. Enf Clinica. 2019;29(S2):413-17. doi: https://doi.org/10.1016/j.enfcli.2019.04.060

31. Chong L, Abdullah A. Community palliative care nurses' challenges and coping strategies on delivering homebased pediatric palliative care: a qualitative study. Am J Hosp Palliat Med. 2017;34(2):125-31. doi: https://doi. org/10.1177/1049909115607296

32. Danielsen BV, Sand AM, Rosland JH, et al. Experiences and challenges of home care nurses and general practitioners in home-based palliative care - a qualitative study. BMC Palliat Care. 2018;17(1):95. doi: https://doi. org/10.1186/s12904-018-0350-0

33. Gágyor I, Heßling A, Heim S, et al. Ethical challenges in primary care: a focus group study with general practitioners, nurses and informal caregivers. Fam Pract. 2019;36(2):22530. doi: https://doi.org/10.1093/fampra/cmy060

34. Yamagishi A, Morita T, Miyashita M, et al. Providing palliative care for cancer patients: the views and exposure of community general practitioners and district nurses in Japan. J Pain Symptom Manage. 2012;43(1):59-67. doi: https://doi.org/10.1016/j.jpainsymman.2011.03.012

35. Costa AP, Poles K, Silva AE. Formação em cuidados paliativos: experiência de alunos de medicina e enfermagem. Interface. 2016;20(59):1041-52. doi: https://doi.org/10.1590/1807-57622015.0774

36. Bellaguarda MLR, Knihs NS, Canever BP, et al. Realistic simulation as a teaching tool in critical situation communication in palliative care. Esc Anna Nery. 2020;24(3):e20190271. doi: https://doi. org/10.1590/2177-9465-ean-2019-0271

37. Cardoso AC, Noguez PT, Oliveira SG, et al. Rede de apoio e sustentação dos cuidadores familiares de pacientes em cuidados paliativos no domicílio. Enferm em foco. 2019;10(3):70-75. doi: https://doi.org/10.21675/2357707X.2019.v10.n3.1792 
38. Oliveira PP, Miranda CE, Lima EHO, et al. Adolescentes em cuidados paliativos: um estudo fundamentado na teoria de Callista Roy. Rev Enferm UFPE on line. 2017;11(Supl. 12):5163-76. doi: https://doi.org/10.5205/1981-8963v11i12a22604p5163-5176-2017

39. Borba JCQ, Zaccara AAL, Andrade FF, et al. Patients at the end of life receiving palliative care: experiences of a multiprofessional team. Rev Fun Care Online. 2020;12:1227-32. doi: http://doi.org/10.9789/21755361.rpcfo.v12.9453

40. Ramalho MNA, Silva LB, Mangueira SO, et al. Cuidados paliativos: percepção de familiares cuidadores de pessoas com câncer. Cienc Cuid Saúde. 2018;17(2):2-6. doi: https://doi.org/10.4025/cienccuidsaude.v17i2

41. Lokker ME, Swart SJ, Rietjens JAC, et al. Palliative sedation and moral distress: a qualitative study of nurses. Appl Nurs Res. 2018;40:157-61. doi: https:// doi.org/10.1016/j.apnr.2018.02.002

42. Aguiar FC, Mendes VLPS. Acreditação hospitalar: a importância da comunicação e da informação para a segurança do paciente. Rev Baiana Saúde Pública. 2016;40(Supl. 1):202-216. doi: https://doi. org/10.22278/2318-2660.2016.v40.n0.a2676

43. Souza EEF, Nevesa LML, Gregório APA, et al. Comunicação de más notícias nos cuidados paliativos oncológicos: revisão integrativa. Diversitates. 2018;10(3):35-47. doi: https://doi.org/10.53357/ EWBA1927 